\title{
1 Evolution of sperm morphology in Daphnia
}

2

3 Duneau David ${ }^{1,4}$, Markus Möst ${ }^{2}$, Dieter Ebert ${ }^{3}$

4

51 Université Toulouse 3 Paul Sabatier, CNRS, IRD; UMR5174, EDB (Laboratoire Évolution \&

6 Diversité Biologique); Toulouse, France

$7 \quad{ }^{2}$ University of Innsbruck, Department of Ecology, Technikerstrasse 25, 6020 Innsbruck, Austria

$8{ }^{3}$ University of Basel, Department of Environmental Sciences, Zoology, Vesalgasse 1, 4051 Basel,

$9 \quad$ Switzerland

$10 \quad{ }^{4}$ Corresponding author: david.duneau@gmail.com 


\section{Abstract}

12 Sperm, the male most fundamental reproductive feature allowing egg fertilization, evolves under

13 sexual selection. Two components of sperm are mainly under selection, their number and their

14 morphology (including the associated quality). Here we study the evolution of sperm morphology in

15 the genus Daphnia. Based on microscopic observations of sperm morphologies mapped on a Daphnia

16 phylogeny, we found that increase in sperm length evolved at least twice in Daphnia, once in the

17 Daphnia sensus lato clade and once in the Ctenodaphnia clade. Furthermore, Daphnia s.l. lost the

18 ability of cell compaction by losing extracellular encapsulation and exposing large filaments. We

19 discuss the potential reasons for such convergent evolution in sperm morphology. 


\section{Introduction}

Sexual selection is a form of natural selection acting on mating and fertilization success. Hence, sperm, the most fundamental male reproductive feature allowing egg fertilization, evolve-at least in part-under such selection. Two components of sperm are mainly under selection, their number and their morphology, the later including the associated quality. Males generally release many gametes, the ejaculate, to fertilize a few eggs. The ejaculate size is known to evolve in response to the risk of sperm competition for egg fertilization, to cryptic female choice and to female receptacle size (i.e. the dilution effect) (Roldan, 2019). In contrast, while sperm are considered as one of the most taxonomically diverse and rapidly evolving cell types (Birkhead et al., 2009; Ramm et al., 2014), the understanding of the adaptive value of sperm morphology, such as length and shape, remains largely incomplete (Lüpold \& Pitnick, 2018). Sperm length does not necessarily correlate with increased swimming speed (although it is often the case, see (Tourmente et al., 2011; Rowley et al., 2019)) and structures not involved in velocity evolve most probably in response to the environment in which fertilization occurs but the details are rarely, if ever, clear (Lüpold \& Pitnick, 2018).

Daphnia are crustaceans reproducing by cyclical parthenogenesis and as such, egg fertilization is sporadic, but essential for diapause in freezing and drying habitats and for dispersal. Following periods of clonal reproduction, during which females only produce genetically identical daughters, and usually triggered by a change in environmental conditions, some females produce sexual eggs while others produce males. During mating, generally one male, but sometimes more, attach to the female to fertilize eggs which will be laid into the female brood pouch after the male(s) departed (Duneau et al., bioRxiv 2020). The brood pouch is a receptacle formed by the carapace and present on the dorsal side of all Daphnia species receiving either clonal or sexual eggs. For the latter, the cuticular structure of the brood pouch changes to form a protective case which will be released upon molting, creating genetically diverse egg-banks from which future populations can be established. Fertilization takes most likely place in this brood pouch (Duneau et al., bioRxiv 2020). However, although there are recordings of males competing for fertilization in Daphnia magna (Duneau et al., bioRxiv 2020), the extend of sperm competition in this receptacle is unknown. Importantly, 
47 a water current generated by the filtering apparatus oxygenates the eggs in the brood pouch (Seidl et

48 al., 2002), and it is likely that many sperm may be flushed out after the male ejaculated. In this context, males are expected to evolve larger ejaculates. However, assuming finite resources allocated to sperm production, this may come at the cost of sperm length (Immler et al., 2011), an important trait in male competition. Thus, males are likely to evolve persistence traits that allow them to increase the chances of fertilization. Here we investigate how sperm morphology in Daphnia species diverged in a phylogenetic context, presumably as a response to sexual selection.

Although pioneer studies have given key general descriptions to identify the main structures (Delavault \& Berard, 1974; Wingstrand, 1978; Zaffagnini, 1987; Wuerz et al., 2017), only little is known about sperm morphology in Daphnia. All Anomopoda, an infraorder including Daphnia, have a vacuolar type of spermatogenesis (Wingstrand, 1978), i.e. in the testes, the spermatids are enclosed in "private" vacuoles in the nutritive cells and are exocytosed into the testicular lumen after they have decreased strongly in size and matured. After they have compacted, they are generally small, about a few microns. Sperm of D. magna has been more thoroughly studied with recent technology. This Ctenodaphnia species has larger sperm $(\sim 10 \mu \mathrm{m})$ encapsulated by an acellular capsule likely compacting radial arms probably to pack more sperm in the testes (Wuerz et al., 2017). The roles of the capsule and of the filaments that are only visible surrounding the cell within the vacuole with electronic microscopy (Wuerz et al., 2017) are unclear. Based on comparison with other models, it has been proposed that female secretion could dissolve the capsule and the filament could have a role in the fusion between gametes (Wuerz et al., 2017). Here, we used a robust phylogeny of the Daphnidae (Adamowicz et al., 2009; Cornetti et al., 2019) and assessed several species representing major clades within Daphnia to better understand the evolution of sperm morphology in this genus.

\section{Materials and methods}

Male Daphnia were either sampled from female mass cultures in the laboratory, where males are naturally produced as a consequence of high density, or from females exposed to the hormone methyl farnesoate (MF, 40nM final concentration) to induce male production. We induced male 


\section{Results \\ Results}

production for D. hyalina, D. zschokkei, D. mendotea, D. galeata, D. curvirostris and D. dentifera and collected naturally produced males for $D$. similis, D. sinensis, D. lumholtzi, D. carinata, D. magna, D. hispanica, D. dolichocephala, D. barbata, D. longispina, D. pulex and D. pulicaria. Note that D. hyalina and D. zschokkei are now synonymous of D. longispina and should be understood as $D$. longispina 'hyalina' and D. longispina 'zschokkei' (Petrusek et al., 2008), hence we merged them on the same branch in the cladogram.

To collect sperm, we exposed mature males to a $1 \%$ nicotine solution ((-)-Nicotin $162.23 \mathrm{~g} / \mathrm{mol}$, from Carl Roth, Germany) to induce ejaculation as in (Duneau et al., 2012). As only mature spermatozoa are in the testicular lumen (p11 in Wingstrand, 1978; p277 in Zaffagnini, 1987), this method allowed us to describe and measure mature sperm and avoid immature ones. Presence of filaments on the sperm was recorded, but we did not measure their length. Measurements of the longest length of the sperm were performed with ImageJ (v. 1.5i) using photographs taken under phase contrast light at magnification 40x. In species with very small sperm (D. pulex, D. pulicaria, D. dolichocephala and D. barbata) we paid particularly attention that the sperm were just released from the spermiduct to reduce the possibility of degradation or to confuse them with other particles. However, it was challenging to take photographs of them, and the measurement may be less accurate than for the other species. $D$. pulex sperm length is only around $2 \mu \mathrm{m}$ in length (Xu et al., 2015). All sperm were also observed at the moment of release from the ejaculatory opening to verify that their shape corresponds to what was observed later when they settled and were photographed. We also observed sperm morphology in sea water to confirm that osmolarity was not affecting our results. Drawing of male abdomens with the genital papilla were taken from published keys (Benzie, 2005; Popova et al., 2016).

Sperm morphology varied greatly among Daphnia, ranging from about $2 \mu \mathrm{m}$ to at least $20 \mu \mathrm{m}$

(Figure 1 and supplementary figures 1 and 2). There was a clear phylogenetic signal in sperm length across Daphnia, but length clusters are polyphyletic. Based on recent Daphnia phylogenies (Adamowicz et al., 2009; Cornetti et al., 2019) and the microscopic observations of sperm 
morphologies, we found that an increase in sperm length evolved at least twice in Daphnia (Figure 1), once in Daphnia sensus lato and once in Ctenodaphnia. This length variation was probably mostly due to a difference in sperm compaction by an extracellular capsule. We found that clades leading to the $D$. longispina group have lost entirely this capsule, hence leaving long filaments without protection (Figure 2, Supplementary figures 1, 2 and 3).

Ceriodaphnia, our outgroup, have sperm of the vacuolar spermatogenesis type, like Daphnia species and the rest of the infraorder of the Anomopoda. Their sperm have been described, based on electron microscopy, as small (2 to $6 \mu \mathrm{m})$, more or less rod-shaped and strongly compacted in their capsule (Figure 2A and p25-26 in Wingstrand, 1978). This information based on several Ceriodaphnia species allowed to determine the most parsimonious ancestral Daphnia sperm morphology. The Ctenodaphnia group, except for D. dolichocephala and D. barbata who had compacted and small sperm, evolved non-compacted and elongated sperm, several times larger than the ancestral morphology (Figure 1 and 2B). A similar adaptation occurred in Daphnia s. l.. As in (Xu et al., 2015) which reported Daphnia pulex s. str. sperm length, Daphnia from the D. pulex subgroup conserved the small and compacted sperm morphology, while D. curvirostris and sister species from the D. longispina species complex also evolved larger elongated sperm, but not as large as in Ctenodaphnia (Figure 1). Additionally, it seems, that Daphnia species from the D. longispina species complex lost the extracellular capsule compacting the sperm and have long filaments with potentially forked structures (Figure 2C). These filaments can be several times the length of the sperm (not measured here) (Figure 2 and Supplementary figure 3). In the same ejaculate from a single D. longispina s. str. male sperm can have, on each side, either one long filament or many shorter ones (see Figure 2 and Supp. material). It has been argued that ejaculate size increases with female receptacle length to compensate for the dilution effect and that it should be accompanied by a reduction in sperm length. Considering that most Ctenodaphnia are not much larger than Daphnia s. l., it is unlikely that this hypothesis explains the evolution of sperm morphology in Daphnia.

We further compared the shape of the male genital papilla across the Daphnia species, using drawing from published keys (Benzie, 2005; Popova et al., 2016) (Figure 1). Most species have no or a very 
126

127

128

129

inconspicuous papilla, the biggest exception is the papilla structure present in D. magna (Figure 1).

This structure is also found in several species related to D. magna, such as D. atkinsoni, D. bolivari and D. mediterranea, but they are not included in our study (Flössner, 2000; Benzie, 2005).

\section{Discussion}

Our assessment of sperm morphology uncovered clearly structured phylogenetic variation in sperm length and in the presence or absence of long filaments. The reason for this variation is unclear but may be in part explained with the strength and intensity of post-copulatory sexual selection. Strength of sexual selection is a function of how often male ejaculates compete for fertilization, in particular direct sperm competition and sperm competition through cryptic female choice.

The frequency of sex in Daphnia depends on the species and of the environment, being eventually under local adaptation (Roulin et al., 2013). In unstable and short-lived habitats, such as small rockpools or ponds in unstable or strongly seasonal environments, such as deserts and arctic sites, few asexual generations occur before diapause recommences. In stable environments, such as large lakes and ponds in temperate mild climatic regions, many asexual generations may occur before the next sexual generation comes, if it ever comes in a mother's lifetime. Traditionally, the later type received more attention by Daphnia researchers, leading to the wrong impression that sexual reproduction, and thus the occurrence of males, is generally rare. Therefore, it is difficult to estimate the intensity and frequency of sexual selection in the system, especially for each species. In certain populations, males can be periodically abundant, and several males can be found copulating at the same time with a female (Duneau et al., bioRxiv 2020). It is not clear whether fertilization would occur in the brood pouch or in the oviduct. However, the fact that most genital papilla are inconspicuous suggest that fertilization is realized in the brood pouch as the access to the oviduct seems complicated. Thus, cryptic female choice and male-male competition via sperm competition, both likely to shape the evolution of sperm morphology, may occur in this female receptacle.

By ejaculating in the female receptacle, males face the challenge to have their sperm flushed out, a phenomenon which could be a form of cryptic female choice. To provide oxygen, the brood pouch is 
152

continuously flushed with a stream of water, entering from the caudal end and leaving from the ventral carapace chamber in a pulsed manner resulting from the rhythmical movements of the limbs (Seidl et al., 2002). This stream might flush out sperm. It is likely that this mechanism selects for males able to produce a large number of sperm of good quality. Interspecies variations in such cryptic female choice may or not vary among species, but males in each species may have different features to increase their chance to be chosen. Sperm may attach and cover the inner lining of the brood pouch, so eggs would not be expelled and touch the sperm as soon as they are released in the brood pouch. However, there is no obvious structure supporting that freshly ejaculated sperm can attach to the inner lining. Then, they may simply increase the number of sperm per ejaculate to increase the chance for few sperm to remain in the brood pouch. However, as most sperm production is done early in male's life (Wuerz et al., 2017) the total number of sperm is limited by the size of the spermiduct. By limiting the total number of sperm stored, this constraint may put selection on the amount of compaction allowing to store more cells in the duct. In Daphnia, there is an extracellular compaction process by an extracellular vacuole before the mature sperm is released into the spermiduct (Wingstrand, 1978). We propose that the evolutionary changes in sperm length we observed may in part be due to a change in the mechanism of cell compaction before maturation.

Female cryptic choice may select for higher number hence smaller sperm, but sperm of different males may also compete within the receptacle imposing an additional selection pressure on sperm morphology. Such sexual selection on sperm morphology through male-male competition will increase as males are more numerous at a given time and regularly mate at the same time with a female. The change in sperm size as a result of sperm competition is a commonly seen evolutionary pattern (see for an example Vielle et al. (2016), including extreme examples like giant Drosophila sperm (Lüpold \& Pitnick, 2018). However, it is difficult to assess the intensity of sperm competition based solely on sperm size. In Caenorhabditis elegans, experimentally enhanced sperm competition leads to the evolution of larger sperm (LaMunyon \& Ward, 2002). However, in Drosophila melanogaster, the competitive advantage was present only in females with relatively long sperm storage organs (Miller \& Pitnick, 2003). When sperm are non-motile, the outcome of sperm 
competition generally resembles a lottery in which having more tickets than your competitor increases your chances to win, males are thus expected to invest in sperm production. Assuming finite resources allocated to sperm production, this may then come at the cost of sperm size (Immler et al., 2011). When sperm competition is high, the total number of sperm may be maximized balancing the size of the sperm per se and its compaction. However, when sperm competition is low, males may invest less in sperm size and there may be little benefit from expending energy in mechanisms insuring constant sperm length (Bauer \& Breed, 2006; Immler et al., 2008). However, because it often correlates with velocity, increase in sperm size can also be selected if sperm are motile at some point of the fertilization process. It is difficult to assure that Daphnia sperm are always non motile. Sperm in the brood pouch are probably not motile but we observed them on a microscope slide and an in vivo assessment would be necessary to ascertain sperm behavior (Lüpold \& Pitnick, 2018). Daphnia males ejaculate into the brood pouch filled with water (the same used for our observation); it is therefore likely that the absence of motility observed in water on a microscope slide reflects what is happening in the brood pouch. However, it has been shown that fluid surrounding eggs of externally fertilized species induced modification to sperm motility (Yoshida et al., 2013). In such scenario, the capsule might break, the sperm would attach to the oocyte, crawl to an eventual specific fusion site and larger sperm with filaments may be advantageous in the race to this site.

Our phylogenetic analysis of sperm morphology revealed a monophyletic clade (i.e. D. longispina species complex) with sperm exposing filaments of diverse shape and length. In Daphnia magna, it seems that filaments exist and are inside the extracellular vacuole (Wuerz et al., 2017), but they are difficult to resolve even with electron microscopy and therefore our knowledge about their ultrastructure is limited. Nevertheless, the vacuole is expected to break before the sperm cell fuse with the oocyte and filaments to be exposed. Daphnia species from the D. longispina species complex evolved non-compacted sperm and the apparent loss of the capsule exposes especially long, sometimes numerous, filaments. Those exaggerated long structures are unlikely to be flagella and not used to move towards the eggs as they are extremely flexible and can be forked. But, as sperm features are expected to be adaptations to their specific fertilization environment (Pitnick et al., 2009), filaments 
206

207

208

209

210

211

212

213

214

215

216

217

218

219

220

221

222

223

almost certainly have a role in fertilization. If we cannot not exclude that they have a role in attachment to the brood pouch to avoid being flushed out with the water flow generated by the female, it is very likely that they have a role in the fusion with the oocyte and potentially with crawling at its surface to reach a fusion site and win the eventual sperm competition. It may be that with reduced selection for compaction, readiness to fuse with the oocyte may be favored by the exposure of those long filaments.

As it is often the case when studying the evolution of sperm morphology, it is difficult to clearly identify the role of sperm features. However, it is certainly evolving driven by the intensity of sexual selection in the system. Daphnia is a cyclical parthenogenetic species, where periods of sexual reproduction are interspersed with period of asexual reproduction. Cyclical parthenogenetic species, like Daphnia, aphids, and rotifers, alternate sexual and asexual reproduction. The number of asexual generations in between two sexual reproductive events may be highly variable, but this is important because the less frequent sexual reproduction is, the lower the average intensity of selection. Thus, one can wonder how the variation in intensity of sexual selection among cyclical parthenogenetic species influences the evolution in sperm morphology. Here, the missing information on the sexual process in Daphnia species makes difficult to assess the relative role of cryptic female choice and sperm competition but both are likely to play a role in the convergent evolution or maybe, even more interestingly, the conserved evolution of sperm length in Daphnia. Altogether, our results support the idea that sexual selection plays a role in cyclical parthenogenetic invertebrate species and encourage further investigations.

\section{Acknowledgements}

Jürgen Hottinger for help in the laboratory. Jeremias Brand, Vitor Faria, Lukas Schärer and Axel Wiberg for discussions about the study. DD was supported by the French Laboratory of Excellence project 'TULIP' (ANR-10-LABX-41; ANR-11-IDEX-0002-02). MM was supported by an FWF Austrian Science Fund stand-alone project (P29667-B25), DE was supported by a grant from the Swiss National Science Foundation. 


\section{References}

233

Adamowicz, S.J., Petrusek, A., Colbourne, J.K., Hebert, P.D.N. \& Witt, J.D.S. 2009. The scale of divergence: A phylogenetic appraisal of intercontinental allopatric speciation in a passively dispersed freshwater zooplankton genus. Mol. Phylogenet. Evol. 50: 423-436.

Bauer, M. \& Breed, W.G. 2006. Variation of sperm head shape and tail length in a species of Australian hydromyine rodent: The spinifex hopping mouse, Notomys alexis. Reprod. Fertil. Dev. 18: 797-805.

Benzie, J.A.H. 2005. Cladocera: The genus Daphnia (including Daphniopsis). Backhuys Publishers, Leiden, The Netherlands.

Birkhead, T.R., Hosken, D.J. \& Pitnick, S. 2009. Sperm biology: an evolutionary perspective, Academic P. Elsevier.

Cornetti, L., Fields, P.D., Van Damme, K. \& Ebert, D. 2019. A fossil-calibrated phylogenomic analysis of Daphnia and the Daphniidae. Mol. Phylogenet. Evol. 137: 250-262.

Delavault, R. \& Berard, J.J. 1974. Ultrastructural study of spermatogenesis in Daphnia magna Straus (Entomostraca, Branchiopoda, Cladocerae).

Duneau, D., Altermatt, F., Ferdy, J.-B.F., Ben-Ami, F. \& Ebert, D. 2020. Estimation of the propensity for sexual selection in a cyclical parthenogen. bioRxiv, doi: https://doi.org/10.1101/2020.02.05.935148.

Duneau, D., Luijckx, P., Ruder, L.F. \& Ebert, D. 2012. Sex-specific effects of a parasite evolving in a female-biased host population. BMC Biol. 10: 104.

Flössner, D. 2000. Die Haplopoda und Cladocera (ohne Bosminidae) Mitteleuropas. Backhuys Publishers, Leiden, The Netherlands.

Immler, S., Calhim, S. \& Birkhead, T.R. 2008. Increased postcopulatory sexual selection reduces the intramale variation in sperm design. Evolution (N. Y). 62: 1538-1543. 
Immler, S., Pitnick, S., Parker, G.A., Durrant, K.L., Lüpold, S., Calhim, S., et al. 2011. Resolving variation in the reproductive tradeoff between sperm size and number. Proc. Natl. Acad. Sci. U. S. A. 108: 5325-5330.

LaMunyon, C.W. \& Ward, S. 2002. Evolution of larger sperm in response to experimentally increased sperm competition in Caenorhabditis elegans. Proc. R. Soc. B Biol. Sci. 269: 1125-1128.

Lüpold, S. \& Pitnick, S. 2018. Sperm form and function: What do we know about the role of sexual selection? Reproduction 155: R229-R243.

Miller, G.T. \& Pitnick, S. 2003. Functional significance of seminal receptacle length in Drosophila melanogaster. J. Evol. Biol. 16: 114-126.

Petrusek, A., Hobæk, A., Nilssen, J.P., Skage, M., Černý, M., Brede, N., et al. 2008. A taxonomic reappraisal of the European Daphnia longispina complex (Crustacea, Cladocera, Anomopoda).

Pitnick, S., Hosken, D.J. \& Birkhead, T.R. 2009. Sperm morphological diversity. In: Sperm Biology, pp. 69-149.

Popova, E. V., Petrusek, A., Kořínek, V., Mergeay, J., Bekker, E.I., Karabanov, D.P., et al. 2016.

Ramm, S.A., Schärer, L., Ehmcke, J. \& Wistuba, J. 2014. Sperm competition and the evolution of spermatogenesis. Mol. Hum. Reprod. 20: 1169-1179.

Roldan, E.R.S. 2019. Sperm competition and the evolution of sperm form and function in mammals. Reprod. Domest. Anim. 54: 14-21.

Roulin, A.C., Routtu, J., Hall, M.D., Janicke, T., Colson, I., Haag, C.R., et al. 2013. Local adaptation of sex induction in a facultative sexual crustacean: insights from QTL mapping and natural populations of Daphnia magna. Mol. Ecol. 22: 3567-3579. 
selection and the evolution of sperm morphology in sharks. J. Evol. Biol. 32: 1027-1035.

282 Seidl, M.D., Pirow, R. \& Paul, R.J. 2002. Water fleas (Daphnia magna) provide a separate ventilatory mechanism for their brood. Zoology 105: 15-23.

Tourmente, M., Gomendio, M. \& Roldan, E.R.S. 2011. Sperm competition and the evolution of sperm design in mammals. BMC Evol. Biol. 11: 12.

Vielle, A., Callemeyn-Torre, N., Gimond, C., Poullet, N., Gray, J.C., Cutter, A.D., et al. 2016. Convergent evolution of sperm gigantism and the developmental origins of sperm size variability in Caenorhabditis nematodes. Evolution (N. Y). 70: 2485-2503.

Wingstrand, K.G. 1978. Comparative spermatology of the Crustacea Entomostraca; 1, Subclass Branchiopoda. Biol. Skr. 22: 1-67.

Wuerz, M., Huebner, E. \& Huebner, J. 2017. The morphology of the male reproductive system, spermatogenesis and the spermatozoon of Daphnia magna (Crustacea: Branchiopoda). J. Morphol. 278: 1536-1550.

Xu, S., Ackerman, M.S., Long, H., Bright, L., Spitze, K., Ramsdell, J.S., et al. 2015. A male-specific genetic map of the microcrustacean Daphnia pulex based on single-sperm whole-genome

Yoshida, M., Hiradate, Y., Sensui, N., Cosson, J. \& Morisawa, M. 2013. Species-specificity of sperm motility activation and chemotaxis: A study on ascidian species. Biol. Bull. 224: 156-165.

Zaffagnini, F. 1987. Reproduction in Daphnia. In: Daphnia (R. H. Peters \& R. De Bernadi, eds), p. 280. Istituto Italiano di Idrobiologia, Pallanza. 


\section{Figure legends}

303 Figure 1: Evolution of sperm and genital papilla morphologies in Daphniidae. Phylogeny of

304 Daphniidea is modified from Cornetti et al. 2019 and Adamowicz et al. 2009. Purple, pink and green

305 represent respectively the sperm that are small, large with capsule or large with filaments exposed.

306 Drawings represent the genital papilla of the males (from Benzie 2005 and Popova et al. 2016) and

307 indicate the atypical exaggerated structure of $D$. magna. Scale bar represent $0,1 \mathrm{~mm}$. Photographs show

308 an example of sperm for each species. The graph represents the difference in sperm length among

309 males. The mean sperm length was calculated with 2 to 3 ejaculates and is intended to provide a rough

310 sense of the traits.

311 Figure 2: Examples of Daphnia sperm morphologies. A- Ceriodaphnia laticauda, ancestral small and

312 encapsulated elongated sperm (purple color in figure 1). Photo from (Wingstrand, 1978). B- D.

313 magna, large and encapsulated elongated sperm of the groups (salmon color in figure 1); C and D - D.

314 longispina, two examples of typical sperm non encapsulated and elongated sperm with filaments

315 (green color in figure 1). 

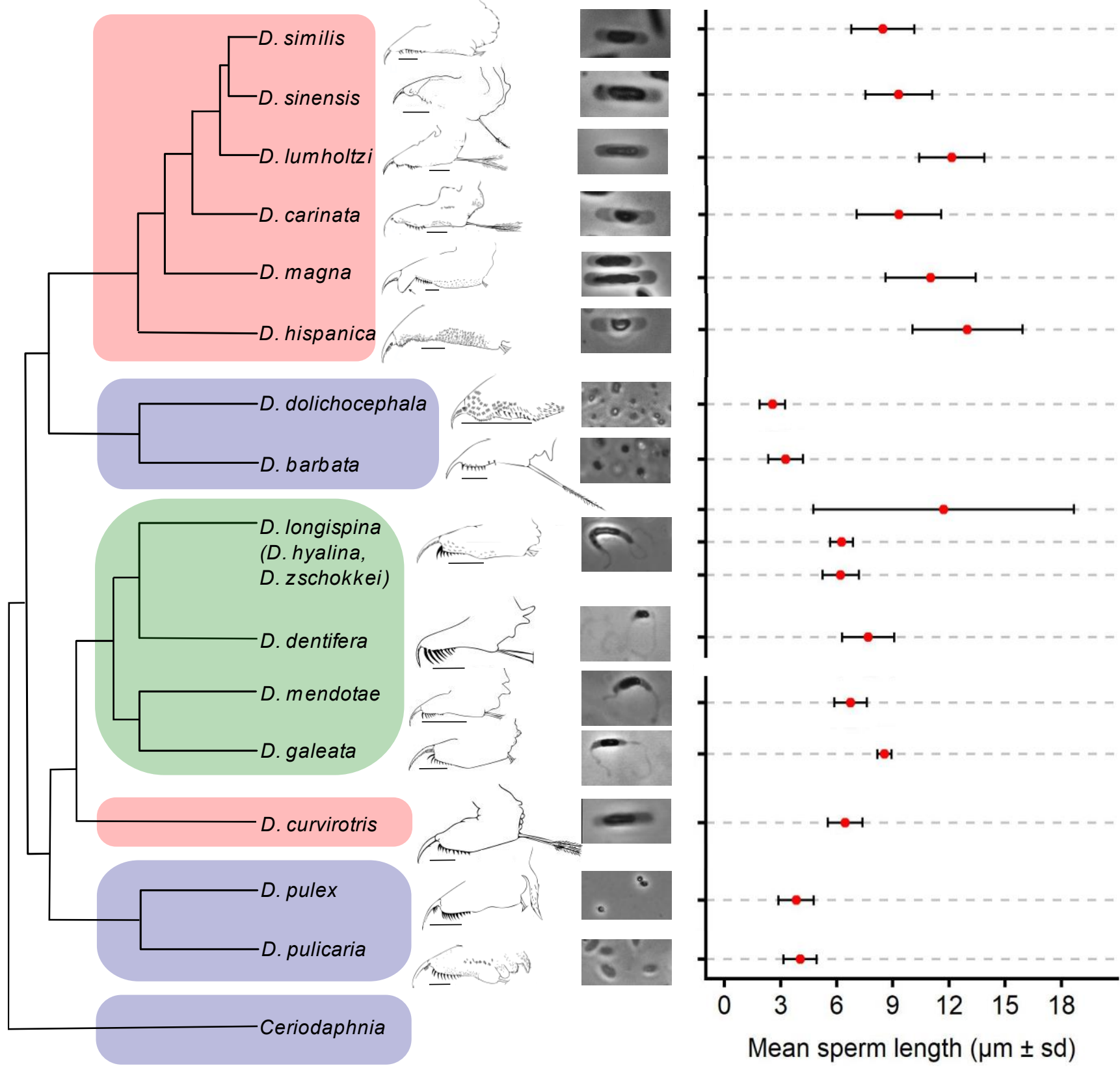

Figure 1: Evolution of sperm and genital papilla morphologies in Daphniidae. Phylogeny of Daphniidea is modified from Cornetti et al. 2019 and Adamowicz et al. 2009. Purple, pink and green represent respectively the sperm that are small, large with capsule or large with filaments exposed. Drawings represent the genital papilla of the males (from Benzie 2005 and Popova et al. 2016) and indicate the atypical exaggerated structure of D. magna. Scale bar represent $0,1 \mathrm{~mm}$. Photographs show an example of sperm for each species. The graph represents the difference in sperm length among males. The mean sperm length was calculated with 2 to 3 ejaculates and is intended to provide a rough sense of the traits. 

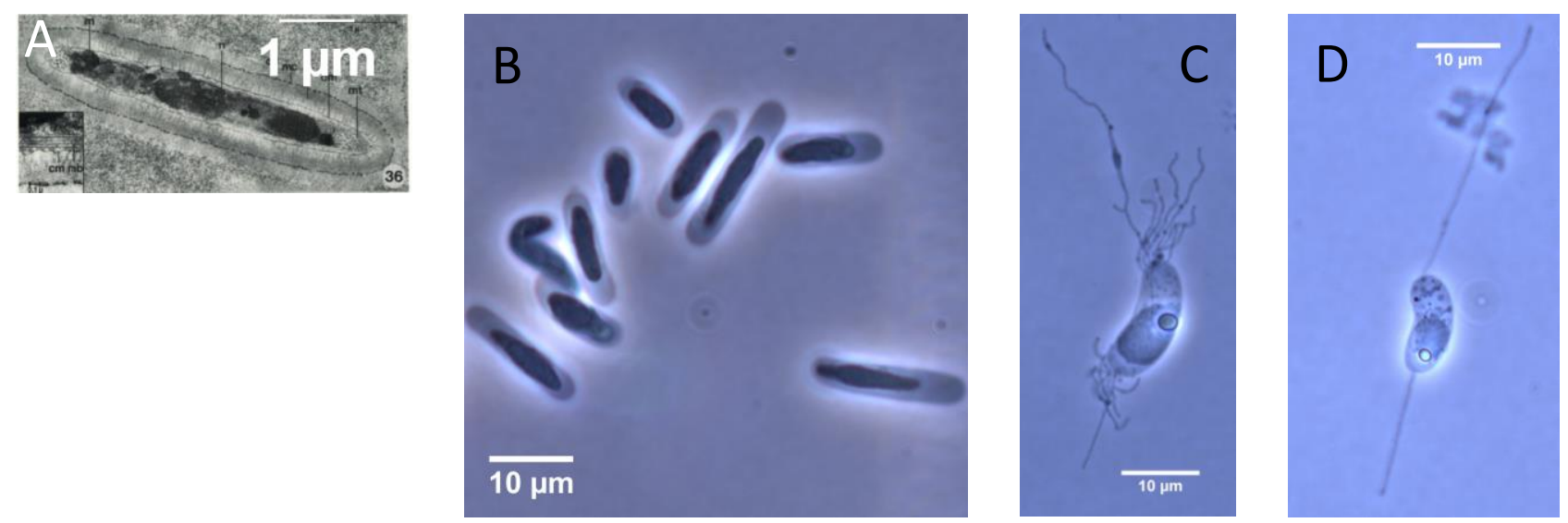

Figure 2: Examples of Daphnia sperm morphologies. A- Ceriodaphnia laticauda, ancestral small and encapsulated elongated sperm (purple color in figure 1). Photo from (Wingstrand, 1978). B- D. magna, large and encapsulated elongated sperm of the groups (salmon color in figure 1); C and D - D. longispina, two examples of typical sperm non encapsulated and elongated sperm with filaments (green color in figure 1). 